\title{
Neuropsychiatric Aspects of Schizophrenia
}

\author{
Raquel E. Gur \\ Departments of Psychiatry, Neurology, \& Radiology, University of Pennsylvania Medical Center, Philadelphia, PA, USA
}

\author{
Keywords \\ Cognitive deficits; Genomic; \\ Neuropsychopharmacology; Schizophrenia.

\section{Correspondence} \\ Raquel E. Gur, Department of Psychiatry, \\ Neuropsychiatry Section, University of \\ Pennsylvania Medical Center, 10th Floor Gates \\ Building, Philadelphia, PA 19104, USA. \\ Tel.: 215-662-2915; \\ Fax: 215-662-7903; \\ E-mail: raquel@upenn.edu
}

doi: $10.1111 / j .1755-5949.2010 .00220 . x$

\begin{abstract}
SUMMARY
Cognitive and affective deficits are prominent clinical features of schizophrenia that impact functioning and are a challenge to effective treatment. The integration of functional magnetic resonance imaging (fMRI) with cognitive and affective neuroscience paradigms enables examination of the brain systems underlying domain-specific behavioral deficits manifested in schizophrenia. There has been a marked increase in the number of studies that apply fMRI in neurobiological studies of schizophrenia. This article highlights phenotypic features of schizophrenia that emerge from these studies and provides a neuropsychiatric perspective. Such efforts have helped elucidate potential neural substrates of deficits in cognition and affect in schizophrenia by providing measures of activation to neurobehavioral probes and connectivity among brain regions. Studies have demonstrated abnormalities at early stages of sensory processing that may influence downstream abnormalities in more complex evaluative functioning. The methodology can help bridge integration with neuropharmacologic, genomic, and neurorehabilitation investigations.
\end{abstract}

\section{Introduction}

Translational efforts in schizophrenia research incorporate diverse methodologies and multiple levels of analysis. They present both opportunities and challenges. The opportunities build on methodological advances including neurobehavioral, neuroimaging, electrophysiology, animal paradigms, genomics, and cellular molecular. The challenges are to advance the understanding of schizophrenia by focused efforts to build the bridge across these disciplines that is necessary to elucidate underlying brain systems. However, when the clinical disorder is heterogeneous, impacts multiple domains, has a lifespan effect and is a treatment challenge; how do we proceed to zoom in? This article highlights the process of transversing the path from examining the phenotypic features of schizophrenia to efforts to investigate the underlying neurobiology.

A neuropsychiatric perspective on schizophrenia examines the phenotypic features of this complex disorder from the viewpoint that aberrant neuronal processes underlie the manifested behavior in affected individuals. This standpoint is not new. Early on schizophrenia has been conceptualized as dementia praecox [1], where deterioration in cognitive abilities was evident in young adults. Similarly, disturbed affect was recognized as a key feature of the disorder [2]. The development and application of neuropsychological tests for assessment of patients with brain lesions provided tools to evaluate the nature and degree of cognitive deficits in schizophrenia. The exciting addition of neuroimaging has fu- eled the field of cognitive and affective neuroscience and has augmented the study of brain function in schizophrenia.

Deficits in cognition and emotion processing constitute major phenotypic features of schizophrenia. Notably, cognition and affect can be studies in neuroimaging paradigms, in animal experiments that model specific behavioral domains and in genetic studies as endophenotypes. Furthermore, they are related to clinical outcome and have treatment implications. Therefore, it is now feasible to construct a neuropsychiatric perspective that elucidates the relation between brain abnormalities, deficits in cognitive and affect processing, and severity of symptoms and functioning related to such deficits.

\section{Cognition in Schizophrenia}

Schizophrenia has been characterized by its apparently dementing features, yet to a large extent the focus of clinical evaluation and intervention has been on the positive symptoms associated with the disorder such as hallucinations and delusions. As psychotic features may interfere in daily functioning in the community, efforts to reduce them are paramount. However, difficulties in the ability to attend, learn, remember, problem solve, and navigate through life, even when psychotic symptoms subside, would impair the ability of people with schizophrenia to live independently.

A large body of literature demonstrates marked deficits in multiple neurocognitive domains that are pertinent for productive 


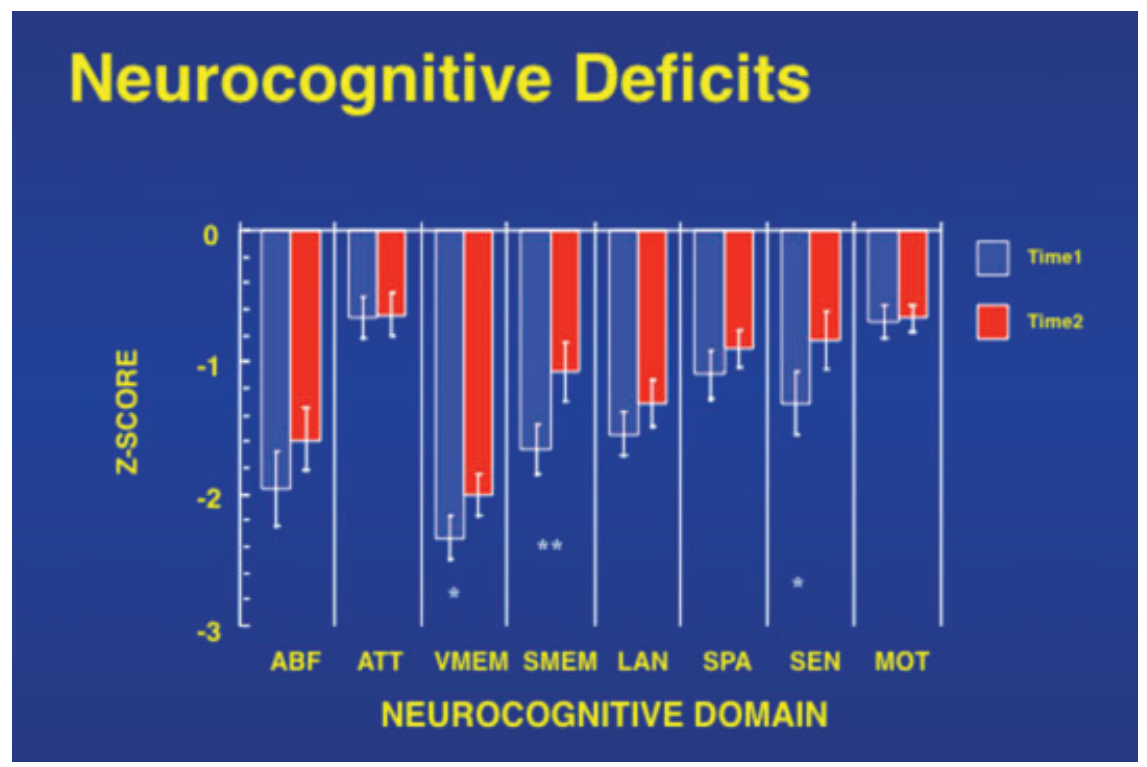

Figure 1 The profile of neurocognitive deficits in schizophrenia at intake (Time 1) and 18 months follow-up (Time 2). ABF, abstraction and mental flexibility; ATT, attention; VMEM, verbal memory; LAN, language; SPA, spatial processing; SEN, sensory; MOT, motor $* P<0.05^{* *} P<0.01$.

functioning. Diffuse deficits have been documented including perception, attention, visuospatial abilities, language, memory, and executive function $[3,4]$. However, within this diffuse impairment, attention, working and episodic memory, and executive functioning are more severely affected [5-7]. While neurocognitive measures have shown limited, albeit consistent, relation to clinical symptoms, they appear more closely related to functional outcome $[8,9]$. These core features of schizophrenia are evident in patients who do not have any other medical, neurological, or psychiatric disorder but schizophrenia, and are important for elucidating underlying mechanisms. Their centrality to schizophrenia of neurocognitive deficits is buttressed by their prominence at initial clinical presentation $[10,11]$, limited amelioration with positive symptom reduction $[4,12]$, link to functional outcome $[8,13]$, evidence for abnormalities in underlying brain circuitry [14-16], and utility as endophenotypes in genetic studies $[17,18]$. Figure 1 presents data from a sample of well-characterized patients with schizophrenia who were followed at the Penn Schizophrenia Research Center. While positive symptom improved over the course of treatment with antipsychotics the cognitive deficits maintained.

The persistence of neurocognitive impairments in schizophrenia has stimulated the investigation of their underlying neurobiology, with the goal of advancing novel interventions. Several brain systems are implicated by these deficits. The attention processing circuitry includes brainstem-thalamo-striato-accumbens-temporalhippocampal-prefrontal-parietal regions. Deficits in working memory implicate the dorsolateral prefrontal cortex, and the ventromedial temporal lobe is implicated by deficits in episodic memory. A dorsolateral-medial-orbital prefrontal cortical circuit mediates executive functions. The application of neurobehavioral probes with functional magnetic resonance imaging (fMRI) provides a powerful method for exploring the neural circuitry un- derlying the observed deficits. In this paradigm participants are engaged in a task while brain activity is measured. This is analogous to a stress test in cardiology, where cardiac parameters are measured during physical activity. In the case of schizophrenia, we evaluate the degree and pattern of brain activity in relation to performance on specific tasks. This application adds to the large literature on MRI neuroanatomic differences evident in people with schizophrenia $[19,20]$.

There has been a marked increase in fMRI studies in schizophrenia that examine the pattern of brain activity in response to neurobehavioral tasks. Failure of patients to activate a specific region indicates difficulties in recruiting the requisite circuitry for that behavioral domain. Such studies have demonstrated abnormal activity in diverse tasks including those related to executive functions such as attention, working memory, and mental flexibility [21-23]. Thus, abnormal activations in ventromedial and superior temporal lobe, prefrontal cortices, and some limbic structures were documented with executive tasks. Notably, it is possible that such complex processes could be compromised as downstream effects of sensory integration deficits that occur early on in the informationprocessing cascade.

To illustrate how such hypotheses can be pursued in fMRI research, the domain of attention will be used as an example. Clinically, people with schizophrenia seem distractable and have difficulties focusing on the task at hand. Rather than shutout nonpertinent stimulation from the environment and fully engaged in the task at end, they seem distracted by irrelevant stimuli and this interferes with their ability to fully attend to what they need to do. A well-replicated body of literatures that examines in the laboratory attentional processes indicates that attentional deficits indeed characterize schizophrenia [24]. Performing attention tasks in the scanner enables to investigate how the brain response of people 


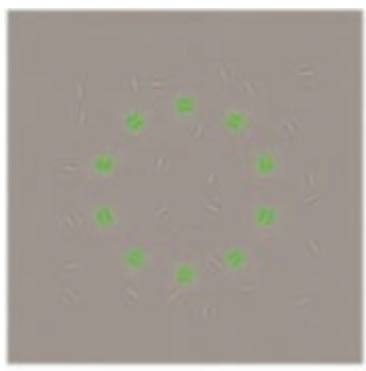

Target

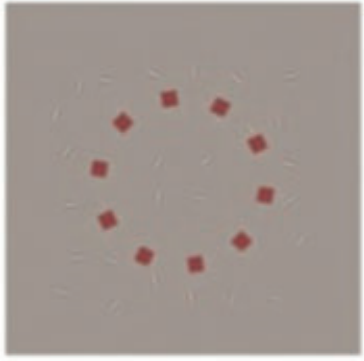

Standard

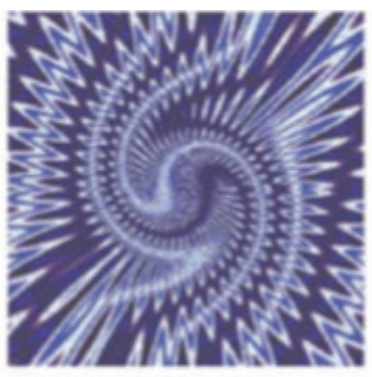

Novel

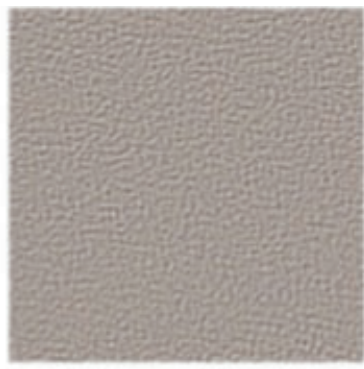

Background

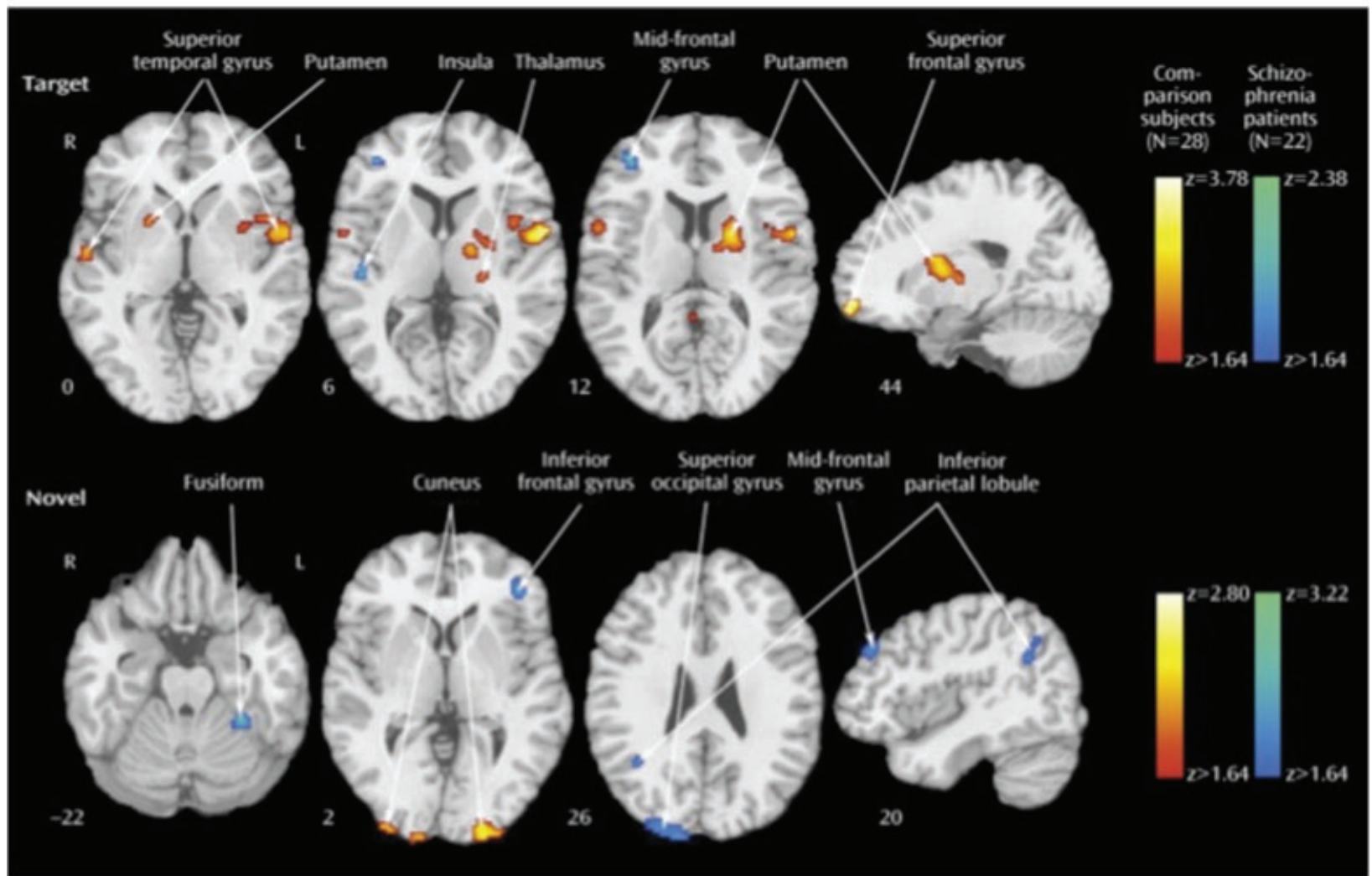

Figure 2 Examples of stimuli used in "oddball" studies of attentional processing (top) and contrast images of patients with schizophrenia and comparison subjects for target and novel stimuli. Greater activation in patients is depicted by the blue scale, whereas greater activation in comparison subjects is shown by the red scale. Images are in radiological convention (left hemisphere to viewer's right). (Reprinted with permission from Gur et al. 2007).

with schizophrenia differs from that of healthy people engaged in an attentional task [25]. Participants were asked to push a button whenever a target appeared instead of a standard stimulus, where no response was required (Figure 2).

To simulate the potential effect of unanticipated additional stimulation during task performance, novel stimuli requiring no response, were presented intermittently. We found that patients and healthy participants showed activation patterns specific to targets and novels, and activation of both neural systems was associated with faster performance. However, reduced activation in regions involved in target processing in patients was accompanied by increased activation in circuits related to elaborated novel stimulus processing. For targets, abnormal activation was noted in regions related to ideational and visual association and for novels patients overactivated sensory and frontal areas related to visual-spatial processing and working memory.

These findings may relate to the attenuated electrophysiological evoked response to targets seen in patients with schizophrenia. Two processes are involved: Insufficient top-down activation of target circuitry and overprocessing of bottom-up events that are distractors. Notably, abnormal activation in patients was associated with more severe clinical symptoms. Thus, in the laboratory setting we can observe that patients are impaired in their ability to direct their attention to the task and at the same time their 
brain responds to unrelated stimuli that they should ignore. This combination jeopardized their ability to attend, which is critical for performing tasks that are necessary for independent functioning.

Another domain that has been examined is verbal learning where deficits are well established in schizophrenia $[6,7,26]$. Patients have difficulties encoding verbal information and perform more poorly than healthy people in how accurately and quickly they can acquire new information. When such tasks are performed in fMRI studies, they have consistently demonstrated abnormalities during the learning phase in fronto-temporal circuits [27,28]. Most studies have reported decreased activation of the frontal cortex, especially the inferior prefrontal region, in schizophrenia. The data are less consistent with regard to the temporal lobe. Most studies have observed decreased activation in patients in the hippocampus and parahippocampal gyrus, but other studies have noted increased activation. Performance may be a contributing factor as the studies differ in their approach to performance. More recently, other fMRI methods have been applied to examine fronto-temporal connectivity and findings suggest that this connectivity is disrupted in schizophrenia. For example, in a study examining within-subject correlations of frontal and temporal timeseries during verbal encoding, aberrant fronto-temporal connectivity was noted in schizophrenia, confirming previous reports [29].

\section{Emotion Processing and Social Cognition}

Impaired emotional functioning is a prominent feature of schizophrenia. Among the negative symptoms of schizophrenia, flat affect has been related to emotion processing. Is reduced emotional expression in schizophrenia related to impaired ability to perceive facial expression of emotions in other people? To address the pertinence of affective flattening, we compared a sample of patients with significant flat affect, assessed by the Scale for Assessment of Negative Symptoms [30] to those without flat affect. Patients with flat affect had poorer premorbid, current, and outcome functioning compared to patients without flat affect [31]. Comparison between the groups in neurocognitive domains indicated that patients with flat affect were more impaired only in verbal memory (Figure 3). Notably, they were also more impaired in emotion identification. Both domains, memory and emotion, are linked to the limbic system.

The behavioral domain that relates to affect and social interactions has been increasingly studied by affective neuroscience paradigms investigating social cognition. Neurobiological studies of social cognition have confirmed that the processing of social information requires complex and synergistic interactions among several neural regions, which progress from basic perception of social stimuli to their later evaluation and ultimate response. Studies have examined brain activity in response to stimuli that differed in emotional valence and in task requirement. For example, there is lack of amygdala activation for sad mood induction in patients with schizophrenia, which are also evident in unaffected siblings [32]. Decreased activation and accuracy in identifying expressions of fear, anger, and disgust, contrasted with responses to mild happiness, was especially evident in patients who were nonparanoid [33]. Presentation of fearful and neutral faces showed

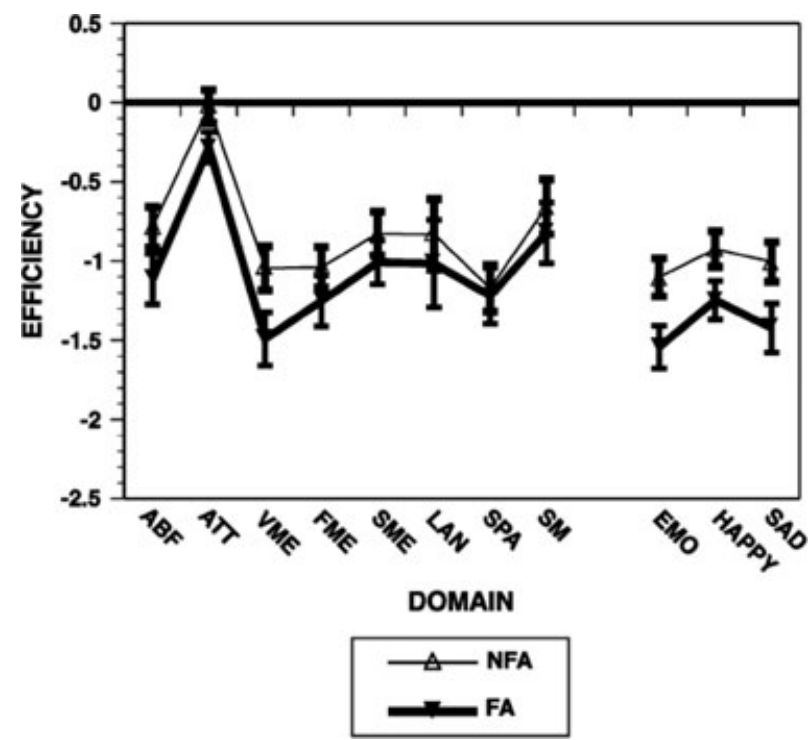

Figure 3 The neurobehavioral profile in patients with flat affect (FA), evaluated by the SANS, and no flat affect (NFA). ABF, abstraction and mental flexibility; ATT, attention; VMEM, verbal memory; FME, face memory; LAN, language; SPA, spatial processing; SM, sensory-motor; EMO, average emotion processing tests. (Reprinted with permission from Gur et al. 2006).

a disconnection between brain activity and arousal in paranoid patients, where increased arousal was associated with decreased amygdala/medial prefrontal activity. In a study contrasting emotion and age identification, patients showed decreased activation in left amygdala and bilateral hippocampus for the emotion condition [34]. In a study examining intensity judgment, patients had an exaggerated amygdala response: positive faces induced right amygdala activation in both groups, while negative faces activated only the right amygdala in controls and bilaterally in patients [35]. Picture categorization (pleasant, unpleasant, neutral) produced less activity in amygdaloid-hippocampal and cortical-basal ganglia-thalamic circuitry in patients [36].

The role of limbic response in identification of facial emotions and its relation to symptoms has been examined in a study that looked both at overall activation for the task of facial affect identification and at activation specifically time-locked to the appearance of threat-related expressions. The results are illustrated in Figure 4 [37].

As can be seen in the middle row, top-down (task related) limbic activation is diminished in schizophrenia. However, patients showed abnormally increased limbic activation time-locked to the appearance of threat-related facial emotions of anger and fear. Furthermore, increased amygdala activation for fear was associated in patients both with failure to identify the emotion and with more severe flat affect (Figure 5).

Studies of emotion processing in schizophrenia vary in methodology and design. Nonetheless, there seems to be considerable convergence of evidence that patients show abnormal activation in amygdala and associated regions. It also appears that the abnormalities are more pronounced for negatively valenced stimuli although no further differentiation for specific emotions has 


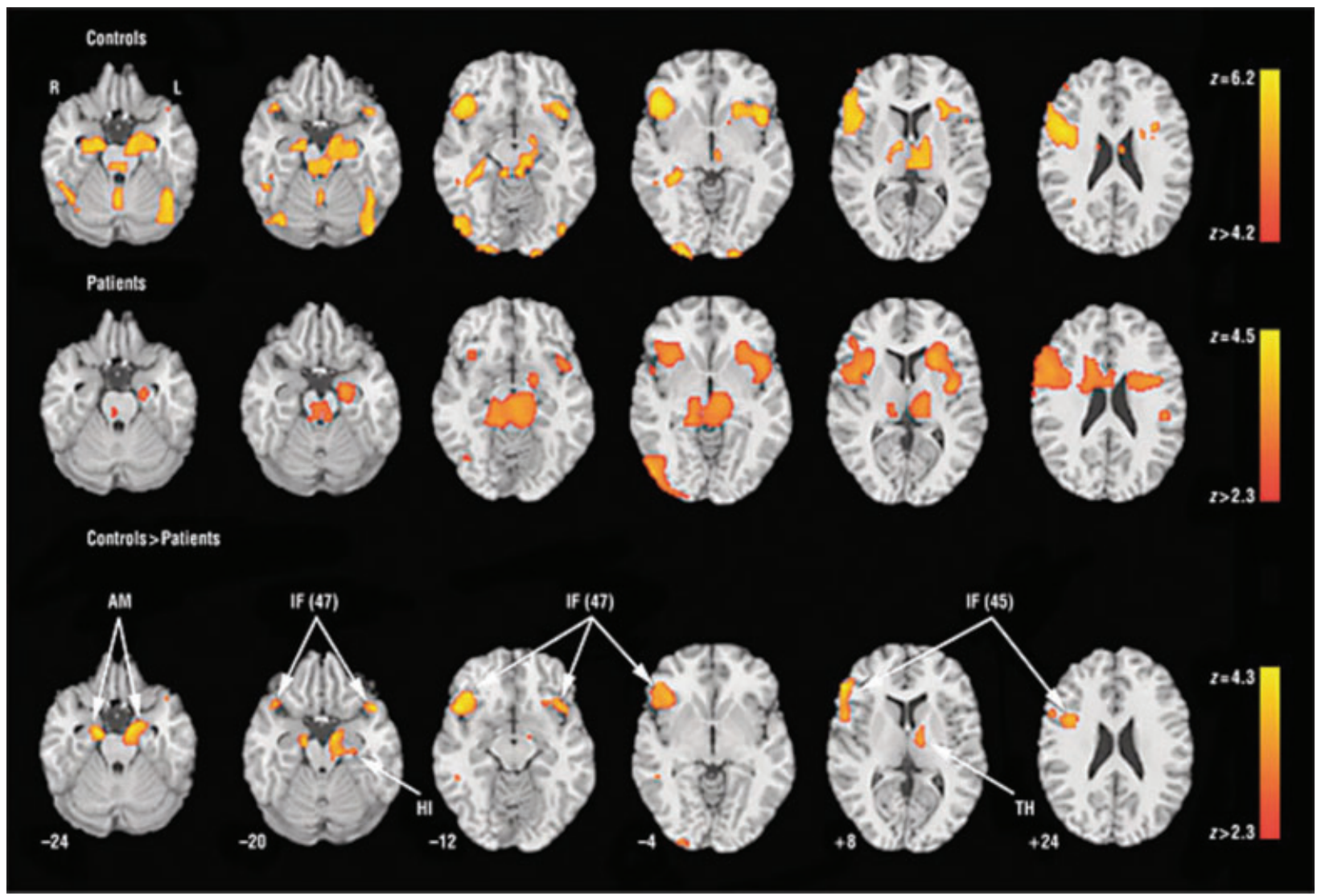

Figure 4 Regions activated for emotion identification task relative to baseline (block analysis) in controls (upper row), patients (middle row), and the controls-patients contrast (bottom row). No patients-controls contrast survived correction. Significance thresholds are based on spatial extent using a height of $z \geq 3.1$ and a cluster probability of $P \leq 0.05$. Images are displayed over a Talairach-normalized template in radiological convention (left hemisphere to viewer's right). The z-level coordinates are provided. AM indicates amygdala; IF (47), inferior frontal (Brodmann area 47); HI, hippocampus; IF (45), inferior frontal (Brodmann area 45); and TH, thalamus. (Reprinted with permission from Gur et al. 2007). been established. In most studies patients' performance was carefully examined and tasks were often constructed so as to minimize performance difference to avoid confounding of physiologic measures. In all cases where performance was evaluated it did not explain the difference in activation patterns. However, reduced taskrelated (top-down) activation could reflect bottom-up interference from abnormally increased amygdala activation related to stimulus valence. Thus, if the task is to identify emotions, sensitivity of the amygdala for specific stimuli may disrupt cortical processes required for categorization and response.

Fronto-temporal connectivity was examined by considering the pattern of correlations among activation parameters obtained from regions recruited for specific tasks. Controls have high specific connectivity for activation to the top-down task and for bottom-up activation associated with correct responding. Patients, by contrast, show abnormally high connectivity associated with activation to threat-related stimuli leading to incorrect responding. Thus, disrupted operation of a top-down process in schizophrenia could be linked to excessive connectivity for bottom-up amygdala activation related to stimulus valence. Such disruptive activation could not only reduce connectivity among regions participating in the top-down process, but also trigger recruitment of other regions, including cortical ones, that may further disrupt task-related processing. Therefore, abnormal amygdala activation will lead to dysfunctional activation in other regions involved in information processing and disrupt downstream processes involving complex behavior. This may manifest in cognitive deficits. A likely specific consequence of abnormal bottom-up activation of amygdala is the disruption of memory processes. There is considerable evidence that the amygdala interacts closely with the hippocampus in the formation of episodic memory, not only when affective valence is prominent. This area merits further investigation.

\section{Summary and Therapeutic Implications}

Considerable advances in cognitive and affective neuroscience, propelled by advances in fMRI methodology, have been applied 


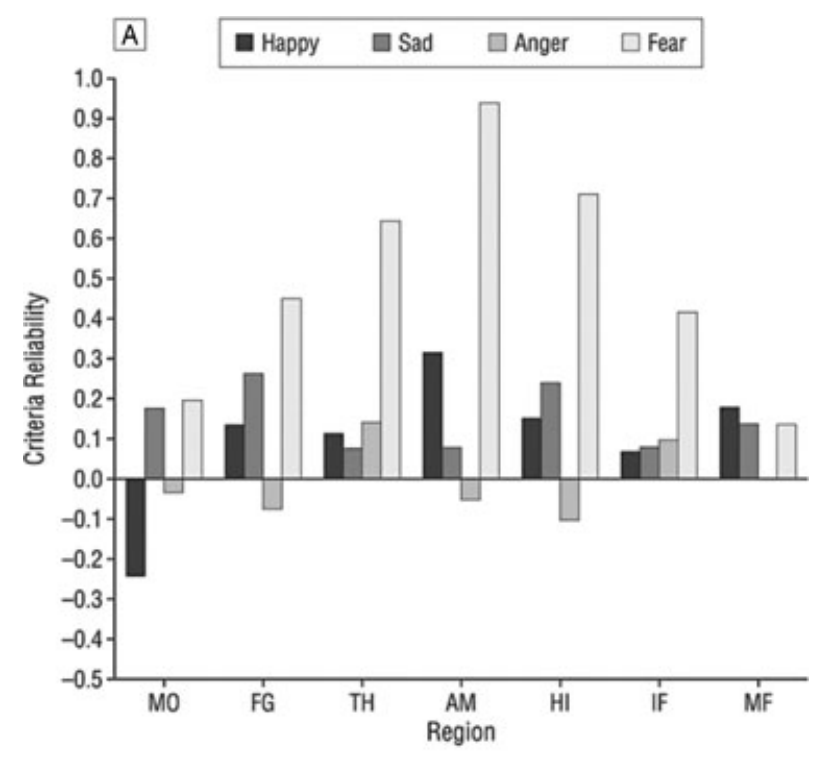

B

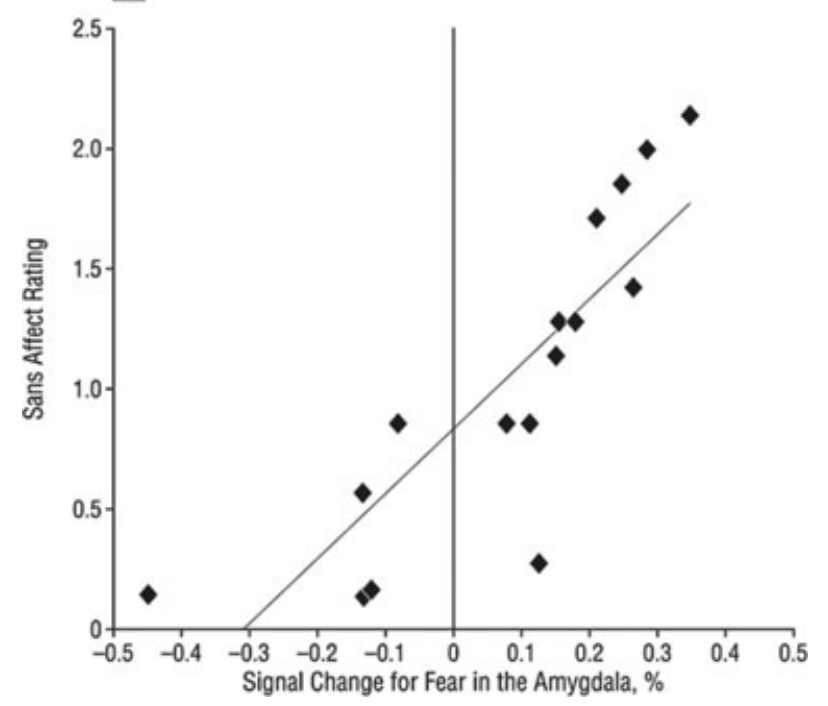

Figure 5 Association between brain activity and clinical measures. (A) Correlations between event-related activation for the four emotional expressions in activated regions and severity of clinical ratings for flat affect. (B) Scatterplot of the association between percentage of signal change for the appearance of fear expressions and severity of flat affect. MO, Midoccipital; FG, fusiform gyrus. Other abbreviations as in Figure 5. (Reprinted with permission from Gur et al. 2007). in efforts to elucidate the neurobiology of schizophrenia. Abnormalities in schizophrenia have been documented at multiple levels of neurobehavioral processing and across several neural systems. These abnormalities are manifested in failure of some regions to activate to a task while other regions overactivate and there are alterations in the connectivity among regions. A clearer picture of these abnormalities and their relation to genetic vulnerability, clinical manifestation, and the potential modulation with treatment will require the combined application of cross-modal measures. The integrative approach enables the construction of a neuropsychiatric perspective of schizophrenia and may inform novel approaches to detection, diagnosis, and treatment.

How advances in clinical and basic neuroscience are translated to the clinical arena is unfolding. In other fields of medicine it is well recognized that early identification of risk factors is necessary to impact illness trajectory. We have seen similar efforts in schizophrenia by identifying subthreshold symptoms in individuals who are at risk for psychosis. The addition of neurobehavioral measures to the clinical assessment enhances the prediction of conversion to schizophrenia. How early interventions, neuropharmaclogical and neurorehabilitative, may alter the course of illness needs to be established.

\section{Acknowledgment}

Supported by NIH grants MH64045 and MH60722.

\section{Disclosures}

Dr. Gur reports investigator-initiated grants from Pfizer and AstraZeneca.

\section{Conflict of Interest}

The authors have no conflict of interest.

\section{References}

1. Kraepelin E. Dementia praecox and paraphrenia. Edinburgh: E. \& S. Livingston, 1919.

2. Bleuler E. Dementia praecox or the group of schizophrenias. New York, NY: International Universities Press, 1950.

3. Heinrichs R. The primacy of cognition in schizophrenia. $A m$ Psychol 2005;60:229-242.

4. Heaton RK, Gladsjo JA, Palmer BW, et al. Stability and course of neuropsychological deficits in schizophrenia. Arch Gen Psychiatry 2001;58:24-32.

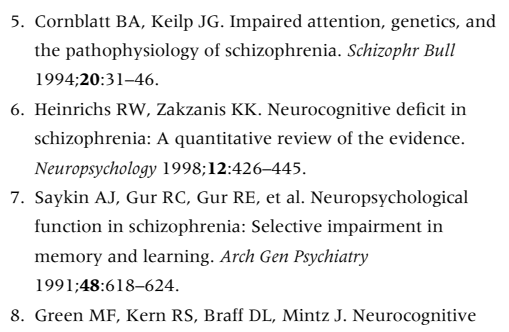

5. Cornblatt BA, Keilp JG. Impaired attention, genetics, and the pathophysiology of schizophrenia. Schizophr Bull 1994;20:31-46.

6. Heinrichs RW, Zakzanis KK. Neurocognitive deficit in schizophrenia: A quantitative review of the evidence. Neuropsychology 1998;12:426-445.

7. Saykin AJ, Gur RC, Gur RE, et al. Neuropsychological function in schizophrenia: Selective impairment in memory and learning. Arch Gen Psychiatry 1991;48:618-624.

8. Green MF, Kern RS, Braff DL, Mintz J. Neurocognitive

deficits and functional outcome in schizophrenia: Are we measuring the "right stuff"? Schizophr Bull 2000;26:119-136.

9. Palmer BW, Dawes SE, Heaton RK. What do we know about neuropsychological aspects of schizophrenia? Neuropsychol Rev 2009; 19:365-384.

10. Saykin AJ, Shtasel DL, Gur RE, et al. Neuropsychological deficits in neuroleptic-naive, first-episode schizophrenic patients. Arch Gen Psychiatry 1994;51:124-131.

11. Zanelli J, Reichenberg A, Morgan K, et al. Specific and generalized neuropsychological deficits: A comparison of 
patients with various first-episode psychosis presentations. Am J Psychiatry 2010;167:78-85.

12. Censits DM, Ragland JD, Gur RC, Gur RE. Neuropsychological evidence supporting a neurodevelopmental model of schizophrenia: A longitudinal study. Schizophr Res 1997;24:289-298.

13. Green MF. What are the functional consequences of neurocognitive deficits in schizophrenia? Am J Psychiatry 1996;153:321-330.

14. Gur RE, Arnold SE. Neurobiology of schizophrenia. In Schatzberg AF, Nemeroff CB, editors. The textbook of psychopharmacology, Vol. 46, 4th ed., Washington D.C.: The American Psychiatric Publishing, 2009;945-963.

15. Calhoun VD, Eichele T, Pearlson G. Functional brain networks in schizophrenia: A review. Front Hum Neurosci 2009;3:1-12.

16. Simpson EH, Kellendonk C, Kandel E. A possible role for the striatum in the pathogenesis of the cognitive symptoms of schizophrenia. Neuron 2010; 65:585-596.

17. Braff DL, Freedman R, Schork NJ, Gottesman II. Deconstructing schizophrenia: An overview of the use of endophenotypes in order to understand a complex disorder. Schizophr Bull 2007;33:21-32.

18. Gur RE, Calkins ME, Gur RC, et al. The consortium on the genetics of schizophrenia: Neurocognitive endophenotypes. Schizophr Bull 2007;33:49-68.

19. Shenton ME, Dickey CC, Frumin M, McCarley RW. A review of MRI findings in schizophrenia. Schizophr Res 2001;49:1-52.

20. Gur RE, Keshavan MS, Lawrie SM. Deconstructing psychosis with human brain imaging. Schizophr Bull 2007; 33:921-931.
21. Ford JM, Gray M, Whitfield SL, et al. Acquiring and inhibiting prepotent responses in schizophrenia: Event-related brain potentials and functional magnetic resonance imaging. Arch Gen Psychiatry 2004;61:119-129.

22. Barch DM, Csernansky JG. Abnormal parietal cortex activation during working memory in schizophrenia: Verbal phonological coding disturbances versus domain-general executive dysfunction. Am J Psychiatry 2007;164:1090-1098.

23. Tan HY, Sust S, Buckholtz JW, et al. Dysfunctional prefrontal regional specialization and compensation in schizophrenia. Am J Psychiatry 2006;163:1969-1977.

24. Nuechterlein KH, Pashler HE, Subotnik KL. Translating basic attentional paradigms to schizophrenia research: Reconsidering the nature of the deficits. Dev Psychopathol 2006; 18:831-851

25. Gur RE, Turetsky BI, Loughead J, et al. Visual attention circuitry in schizophrenia investigated with oddball event-related functional magnetic resonance imaging. Am J Psychiatry 2007;164:442-449.

26. Aleman A, Hijman R, de Haan EH, Kahn RS. Memory impairment in schizophrenia: A meta-analysis. Am J Psychiatry 1999;156:1358-1366.

27. Ongür D, Cullen TJ, Wolf DH, Rohan M, Barreira P, Zalesak M, Heckers $S$. The neural basis of relational memory deficits in schizophrenia. Arch Gen Psychiatry 2006;63:356-65

28. Ragland JD, Laird AR, Ranganath C, Blumenfeld RS, Gonzales SM, Glahn DC. Prefrontal activation deficits during episodic memory in schizophrenia. Am J Psychiatry 2009;166:863-874.
29. Wolf DH, Turetsky BI, Loughead J, Elliott MA, Pratiwadi R, Gur RE, Gur RC. Auditory oddball fMRI in schizophrenia: Association of negative symptoms with regional hypoactivation to novel distractors. Brain Imaging Behav 2008;2:132-145.

30. Andreasen NC. The scale for the assessment of negative symptoms (SANS). Iowa City: The University of Iowa, 1984.

31. Gur RE, Kohler CG, Ragland JD, Siegel SJ, Lesko K, Bilker WB, Gur RC Flat affect in schizophrenia: Relation to emotion processing and neurocognitive measures. Schizophr Bull 2006;32:279-287.

32. Habel U, Klein M, Shah NJ, Toni I, Zilles K, Falkai P, Schneider F. Genetic load on amygdala hypofunction during sadness in nonaffected brothers of schizophrenia patients. Am J Psychiatry 2004;161:1806-1813.

33. Phillips ML, Williams $\mathrm{L}$, Senior $\mathrm{C}$, et al. A differential neural response to threatening and non-threatening negative facial expressions in paranoid and non-paranoid schizophrenics. Psychiatry Res 1999;92:11-31.

34. Gur RE, McGrath C, Chan RM, et al. An fMRI study of facial emotion processing in patients with schizophrenia. Am J Psychiatry 2002;159:1992-1999.

35. Kosaka H, Omori M, Murata T, et al. Differential amygdala response during facial recognition in patients with schizophrenia: An fMRI study. Schizophr Res 2002;57:87-95

36. Takahashi H, Koeda M, Oda K, et al. An fMRI study of differential neural response to affective pictures in schizophrenia. Neuroimage 2004;22:1247-1254

37. Gur RE, Loughead J, Kohler CG, et al. Limbic activation associated with misidentification of fearful faces and flat affect in schizophrenia. Arch Gen Psychiatry 2007;64:1356-1366. 